\title{
Nanoscale Resolution in the Focal Plane of an Optical Microscope
}

\author{
Volker Westphal and Stefan W. Hell* \\ Department of NanoBiophotonics, Max Planck Institute for Biophysical Chemistry, 37070 Göttingen, Germany
} (Received 16 November 2004; published 15 April 2005)

Utilizing single fluorescent molecules as probes, we prove the ability of a far-field microscope to attain spatial resolution down to $16 \mathrm{~nm}$ in the focal plane, corresponding to about $1 / 50$ of the employed wavelength. The optical bandwidth expansion by nearly an order of magnitude is realized by a saturated depletion through stimulated emission of the molecular fluorescent state. We demonstrate that en route to the molecular scale, the resolving power increases with the square root of the saturation level, which constitutes a new law regarding the resolution of an emerging class of far-field light microscopes that are not limited by diffraction.

DOI: 10.1103/PhysRevLett.94.143903

The discovery of the diffraction barrier in the 19th century [1] suggested that a far-field light microscope would never be able to resolve objects closer than about $\lambda /(2 n \sin \alpha)$, with $\lambda, n$, and $\alpha$ denoting the wavelength of light, the refractive index, and the semiaperture angle of the lens, respectively. The eminent role of light microscopy in science has turned this notion into one of the most prominent paradigms of physics. Since then, the diffraction barrier has been addressed sporadically, but neither the use of aperture filters [2] nor the advent of nonlinear optics improved the far-field microscopy resolution by more than a factor of 2. In the 1990s, the proposal of stimulated emission depletion (STED) fluorescence microscopy [3] indicated that, at least for fluorescence imaging, it should be possible to attain a spatial resolution of a fraction of $\lambda$. Meanwhile, the quest for nanoscale resolution with focused visible light has become a hot topic. Relying on conventional lenses, far-field microscopy does not require tight control of a tip as is the case with near-field optics. Moreover, it is fast, easy to use, and above all not surface bound, which is essential to many applications, such as the imaging of cells.

An intriguing approach to obtaining far-field resolution at $\lambda /(2 m)$, with $m>1$, is to employ an $m$-photon excitation with $m$ entangled photons [4]. However, this approach is hampered by the lack of bright $m$-entangled photon sources and the fragility of the entanglement. Strongly focused light of radial polarization generates 1.3 times sharper foci, but at the same time also generates higher sidelobes [5]. Subdiffraction resolution has been achieved with STED, but the focal plane resolution remained relatively modest as well $[6,7]$. The combination of STED with 4Pi-microscopy has lead to a $z$ resolution of $30-50 \mathrm{~nm}$ [8]. However, the focal plane $(x y)$ resolution is not improved in this scheme. Moreover, a large part of the improvement in $z$ originated from the coherent counter-propagating waves of the 4Pi-microscope.

Here we report on a focal spot size of $16 \mathrm{~nm}$ in a microscope with a novel implementation of STED, corresponding to $\sim 1 / 50$ of the responsible wavelength. Exceeding previously reported values by far, including
PACS numbers: 42.30.-d, 42.25.-p, 78.45.+h, 87.64.-t

those based on computational enhancement [7], our data evidence for the first time all-physical nanoscale resolution with visible light and conventional lenses. Moreover, we prove experimentally that the minimal resolving distance scales inversely with the square root of the saturation level (of STED). This dependence is established as a new law in far-field optical microscopy resolution, extending Abbe's resolution equation.

Because of diffraction, the minimal size of a fluorescent spot in the focal plane of a far-field scanning microscope is limited to $180 \mathrm{~nm}$ at most. In STED microscopy, molecular excitation is performed with a laser pulse which is then immediately followed by a redshifted one for depleting the fluorescent state by stimulated emission [3,6]. Both pulses are diffraction limited, but the latter, referred to as the STED pulse, is modified to form a central zero, e.g., a focal doughnut. Superposing the two spots confines the emission toward the zero. Specifically, if the intensity of the STED pulse surpasses the threshold required for depletion, the spot from which fluorescence may originate is a minimal area around the zero that decreases with an increase in STED pulse intensity. Scanning this narrowed spot across the sample yields subdiffraction images. Importantly, the concept of STED may be generalized to encompass any reversible saturable optical transition, including nonfluorescent ones, such as a transient saturated population of the molecular triplet state, self-induced optical transparency, or in fact any reversible photoinduced transition between two states [9].

The resolution increase expected from STED is readily derived. Let $h_{\text {exc }}(r)$ be the normalized excitation probability in the focal plane resulting from the excitation pulse. The typical fluorescence and vibrational lifetimes of a dye of $\sim 3 \mathrm{~ns}$ and $0.5 \mathrm{ps}$, respectively, ensure that with a STED pulse duration $\tau$ of $\sim 0.1 \mathrm{~ns}$, the fluorescence is effectively quenched. Hence after the passage of the STED pulse, the fluorescence probability $\eta(r)$ of a molecule has decayed to $\eta(r)=\exp \left[-\sigma \tau I_{\mathrm{STED}}(r)\right]$, with $\sigma$ and $I_{\mathrm{STED}}(r)$ denoting the cross section for stimulated emission and the focal intensity of the STED pulse, respectively. Thus, the probability to detect a photon from $r$ is propor- 
tional to $h(r)=h_{\mathrm{exc}}(r) \eta(r)$. The width of the focal plane maximum of $h_{\text {exc }}(r)$, e.g., in the $x$ direction, has as its lower limit the peak of a standing wave still allowed by diffraction. The same holds for the focal minimum of the STED pulse. Given a lens with semiaperture $\alpha$, we have:

$$
\begin{aligned}
h_{\mathrm{exc}}(r) & =C \cos ^{2}\left(\pi r n \sin \alpha / \lambda_{\mathrm{exc}}\right), \\
I_{\mathrm{STED}}(r) & =\varsigma I_{\mathrm{sat}} \sin ^{2}\left(\pi r n \sin \alpha / \lambda_{\mathrm{STED}}\right) .
\end{aligned}
$$

For $I_{\text {sat }}=1 / \sigma \tau$, the fluorescence has dropped to $1 / e$ of the initial value. Introducing $\max \left[I_{\mathrm{STED}}(r)\right]=I_{\mathrm{STED}}^{\max }$ enables us to define the saturation level of STED as $\varsigma=I_{\text {STED }}^{\max } / I_{\text {sat }}$. With $\lambda_{\text {exc }} \approx \lambda_{\text {STED }} \equiv \lambda$ and $r<\lambda / 2 n$, the full-width-halfmaximum (FWHM) of $h(r)$, i.e., the size of the spot in which fluorescence occurs, is approximated by a Taylor series to the second order:

$$
\Delta r=\frac{\sqrt{8}}{\pi \sqrt{1+I_{\mathrm{STED}}^{\max } / I_{\mathrm{sat}}}} \frac{\lambda}{2 n \sin \alpha} \approx 0.45 \frac{\lambda}{n \sin \alpha \sqrt{1+\mathrm{s}}} .
$$

The orientation of the linear molecular transition dipole $(\varphi$, $\vartheta$ ) with respect to the linearly polarized stimulating light field used in this work is readily incorporated in the scalar Eq. (2) by weighting the effective $\sigma$ by $\cos ^{2}(\varphi) \cos ^{2}(\vartheta)$. The angles $\varphi$ and $\vartheta$ are the azimuthal and polar angles formed by the transition dipole with the field component in the focal plane. Excitation of and detection from molecules oriented off the focal plane are favorably suppressed by $\cos ^{4}(\vartheta)$.

Starting from circularly polarized excitation and unpolarized detection (case $A$ ), the photoselection can be augmented by $\cos ^{4}(\varphi)$ by employing collinearly polarized excitation and detection that are parallel to the STED field (case $B$ ). Numerical averaging over many randomly oriented molecules shows that the orientational effects lead to an effective $\varsigma$ of $\varsigma_{A}=0.35 \varsigma$ and $\varsigma_{B}=0.69 \varsigma$, for cases $A$ and $B$, respectively. Whereas $\varsigma=0$ in Eq. (2) largely reproduces Abbe's diffraction limit, for $\varsigma \rightarrow \infty$ the lateral spot width decreases continually, following a square-root law.

To explore this law, we imaged single molecules of the xanthene dye JA26 spin coated on a glass slide in a polyvinyl alcohol (PVA) solution. While the excitation was performed with 68 ps pulses at $\lambda_{\text {exc }}=635 \mathrm{~nm}, 300 \mathrm{ps}$ pulses at $\lambda_{\text {STED }}=775 \mathrm{~nm}$ were used for STED. The $76 \mathrm{MHz}$ pulse trains were collinearly directed towards an oil immersion lens of $\alpha=68^{\circ}$. The fluorescence image was gained through scanning the sample with respect to the stationary beams. Before entering the lens, the wave front of the STED beam was modified to either form a doughnut, or a unidirectional valley squeezing the spot just along the $x$ axis. The latter is produced through phase shifting by $\pi$ half of the STED pulse wave front in such a way that the dividing line is parallel to the $y$ axis [6]. In conjunction with $y$ polarization, destructive interference yields a $y$-oriented valley in the focal region. This line-shaped minimum is readily expanded to two dimensions by further adding an $x$-oriented, $x$-polarized counterpart [Fig. 1(a)]. The composite is a doughnut that is purely $x$ polarized along the $y$ axis and vice versa, but with both polarization components along oblique sections. In the PVA matrix, the transition dipole moment of the molecule is not expected to rotate after excitation. Therefore, in our experiments, the $y$-oriented valley and the composite doughnut were paired with $y$ - and circular-polarized excitation (cases $B$ and $A$ ), respectively.

Figure 1(a) and 1(b) show $x y$ images that have been recorded from the same site. However, in Fig. 1(a), a doughnut with $I_{\text {STED }}^{\max }=800 \mathrm{MW} / \mathrm{cm}^{2}$ and a zero of $<2 \%$ of $I_{\text {STED }}^{\max }$ was used. At this $I_{\text {STED }}^{\max }$, STED was measured to yield $\varsigma_{A}=36$ for randomly distributed JA26 molecules. As a result, the STED image displays distinct spots featuring $\Delta r=30-35 \mathrm{~nm}$ lateral FWHM, exemplifying a sevenfold increase in $x$ and $y$ resolution over the diffraction barrier.

Belonging to single (or a few) molecules, the spots vary in size depending on the orientation of the molecules to the doughnut field. Displaying the $x$ and $y$ FWHM of 90 spots, Fig. 1(d) reveals the hallmark of the doughnut polarization in the distribution of the spot size. While most of the spots are round or just slightly elliptical, some of them are narrowed in the $x$ or $y$ direction only. As the effective s varies with $\cos ^{2}(\varphi)$, unilaterally narrowed spots are anticipated from molecules with vanishing $y$ - or $x$-transition dipole components. This is further illustrated in the insert in Fig. 1(d), displaying $\Delta \eta=\eta_{x}(r)-\eta_{y}(r)$, with $\eta_{x, y}$
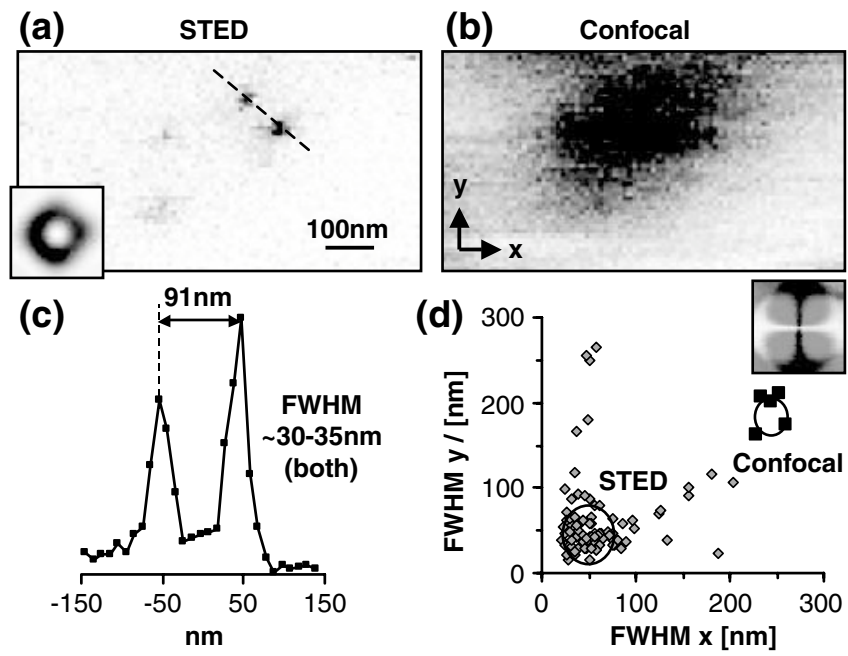

FIG. 1. Far-field fluorescence microscopy with an effective focal area 25 times below the diffraction limit. Comparison between (a) STED and (b) confocal image of molecules on a surface. Insert in (a) shows the STED doughnut employed (not to scale); (c) data profile along the dashed line in (a) evidences 30$35 \mathrm{~nm}$ spot size; (d) observed subdiffraction FWHM of fluorescence spots; circles mark the standard deviation of the respective distributions. Insert in (d) displays the spatial distribution of fluorescence probability after STED calculated as $\eta_{x}-\eta_{y}$ (explanation in the text). 
(a) Confocal
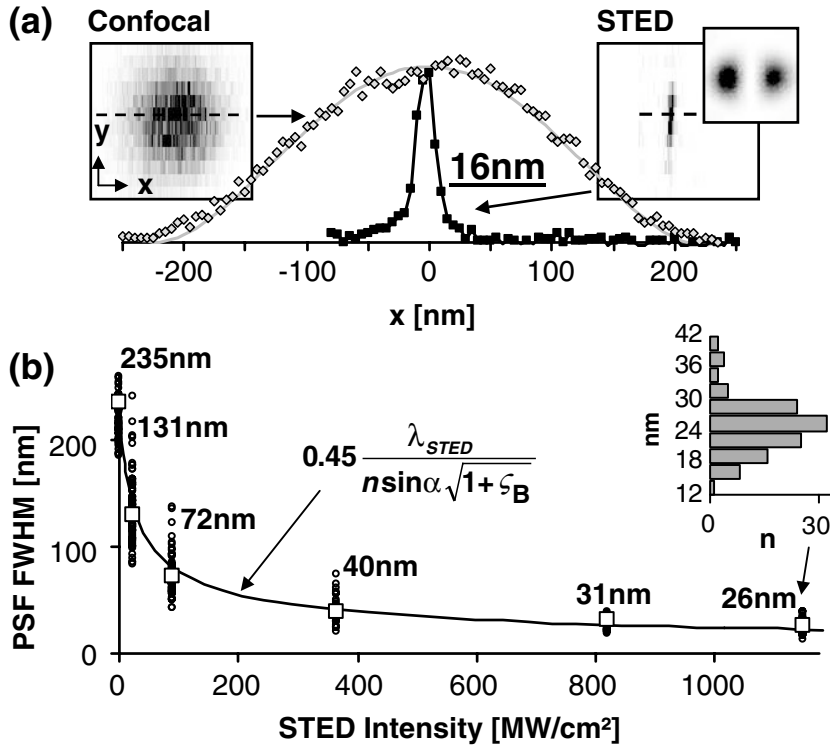

FIG. 2. Reducing the fluorescence focal spot size to far below the diffraction limit: (a) spot of a confocal microscope (left) compared with that in a STED microscope (right) utilizing a $y$-oriented intensity valley for STED (upper right insert, not to scale) squeezing the spot in the $x$ direction to $16 \mathrm{~nm}$ width. (b) The average focal spot size (squares) decreases with the STED intensity following a square-root law, in agreement with Eq. (2). Insert (right) discloses the histogram of the measured spot sizes rendering the $26 \mathrm{~nm}$ average FWHM.

denoting the fluorescence left over by the $x$ - and $y$-polarized components of the doughnut, respectively. For $\Delta \eta \approx 0$ (gray regions) STED is about equally efficient for all molecular orientations, while for $\Delta \eta \gg 0$ (white) and $\Delta \eta \ll 0$ (black), horizontally and vertically oriented molecules are not depleted, respectively. Altogether, when applying this particular doughnut at $\varsigma_{A}=36$, the brightness-weighted average FWHM is 47 and $45 \mathrm{~nm}$ in $x$ and $y$, respectively. Thus, the average focal crosssectional area is reduced by a factor of 25 when compared to the diffraction limit.

To explore the highest possible resolution under this set of conditions, we increased $s$ in two ways. By directing all power available into the narrow $y$-oriented valley, we generated a peak intensity of $1.1 \mathrm{GW} / \mathrm{cm}^{2}$ with a minimum depth of $<1 \%$. The combination with collinearly polarized excitation, STED, and detection (case $B$ ) yielded $S_{B}=112$. The result is a stronger narrowing of the spot in the $x$ direction [Fig. 2(a)]. Whereas the confocal spot features an FWHM of $235 \mathrm{~nm}$, the spot left by STED features an $x$ FWHM of $\Delta x=16 \mathrm{~nm}$.

The profile in Fig. 2(a) is selected from a set of $x$-squeezed spots, which, due to orientational variation of the molecules, range in size from $40 \mathrm{~nm}$ down to $14 \mathrm{~nm}$. The narrowest spots favorably are also the brightest, which results from the fact that the light fields for excitation and stimulated emission are parallel. The average FWHM is
$26 \mathrm{~nm}$ at this $\mathrm{s}$. The full dependence of the spot FWHM on $s$ is displayed in Fig. 2(b), confirming the square-root law predicted by Eq. (2).

We observed that with an increase in STED intensity the fluorescence was slightly depleted at the focal center, although this unintended loss of peak brightness never exceeded 25\%. Likewise, we observed increased bleaching when applying STED, but again its contribution to the bleaching of these molecules was $<50 \%$ of that by the excitation alone.

The subdiffraction resolution is also evident from the optical transfer function (OTF) of the system, calculated as the magnitude of the Fourier transform of the summed response of many individual molecules. Displaying the OTF for both the doughnut and the line-shaped valley, Fig. 3(a) reveals a fivefold and eightfold gain in bandwidth, respectively, over that of confocal microscopy. As a result, molecules that are only a fraction of $\lambda$ apart, e.g., at $45 \mathrm{~nm}$ as in Fig. 3(b) and 3(c), appear as distinct spots in the image. The OTF also determines the bandwidth for optional image processing. For example, an OTF-based linear deconvolution (Wiener filtering) was applied to the righthand single molecule image in Fig. 3(b). While the raw image exhibits a modulation depth of $70 \%$, subsequent linear deconvolution fully discerns molecules at a $45 \mathrm{~nm}$ distance.

Finally, we demonstrate the subdiffraction lateral resolution in a nonsparsely prepared sample. For that purpose, we wetted the surface of an $\mathrm{Al}_{2} \mathrm{O}_{3}$ matrix featuring
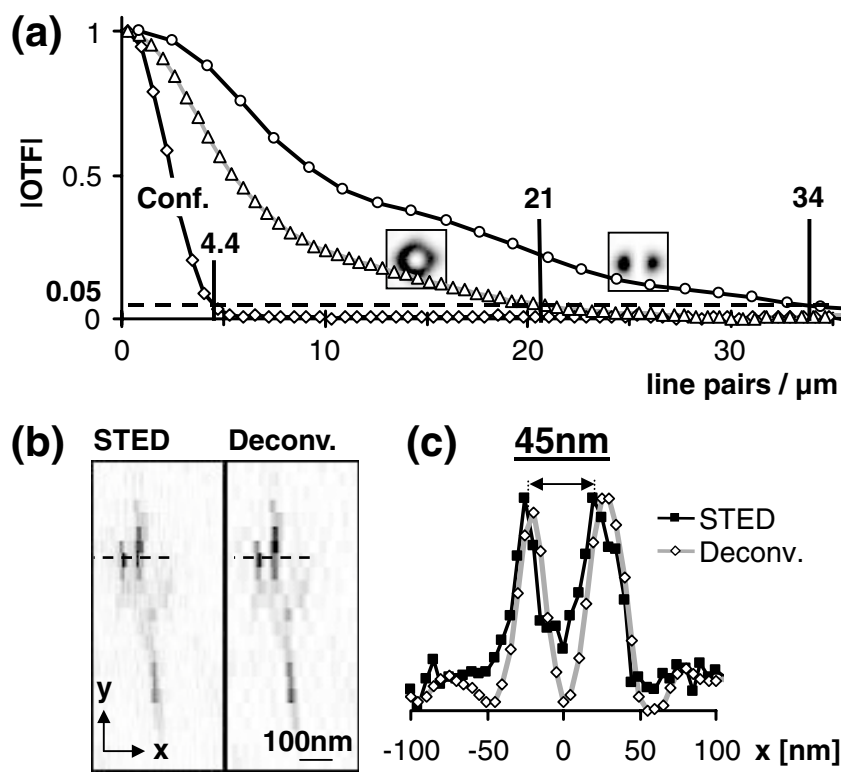

FIG. 3. (a) Fundamental increase of bandwidth in STED (uniand bilateral) over confocal microscopy: normalized magnitude of optical transfer function (OTF) as a function of line pairs per $\mu \mathrm{m}$; (b) $x y$ image of molecules with subdiffraction resolution in $x$ : raw data and post-Wiener filtering data shown in left and right-hand panel, respectively, discerning molecules at $45 \mathrm{~nm}$ distance; (c) profile along dashed line in (b). 


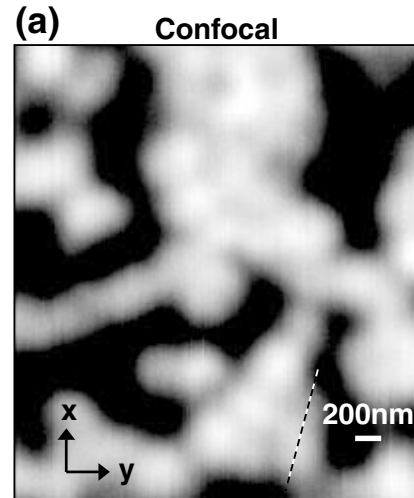

(c)

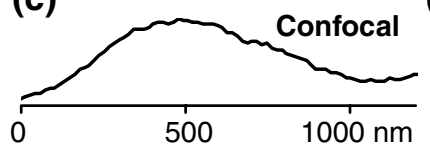

(b)

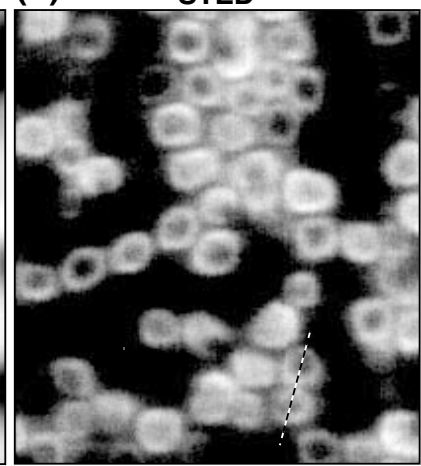

(d)

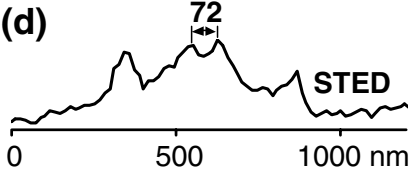

FIG. 4. Subdiffraction fluorescence imaging reveals a complex pattern on a wetted surface: (a) confocal (b) STED microscopy image. (c), (d) Line profiles along dotted lines in (a) and (b).

$z$-oriented holes (Whatman plc, Brentford, UK) with a spin cast of a dyed polymethyl methacrylate solution. The pattern of rings formed in this way was resolved when applying the STED doughnut at $I_{\text {STED }}^{\max }=1 \mathrm{GW} / \mathrm{cm}^{2}$ (Fig. 4). Profiles through the raw image data show that rings located only $72 \mathrm{~nm}$ apart, i.e., $\lambda / 10$, can be separated.

While the slight asymmetric spot squeezing highlighted in Figs. 1-3 corroborates the principles of STED, it must not be perceived as an inherent limitation of the concept itself. Circularly polarized doughnuts will enable symmetrically squeezed spots irrespective of the molecular orientation. Nevertheless, even for the random orientation of the molecules, the comparison image in Fig. 4 displays a marked improvement in resolution.

The validation of Eq. (2) implies that the resolution can be further augmented. Challenges to be met on the way include the stronger photobleaching with larger $s$ and the production of focal zeros $<0.5 \%$. Both issues can probably be addressed in the future.

The nanoscale resolution described in this study is due to the exponential dependence of the fluorescence on the applied intensity (of the STED beam). In contrast to multiphoton nonlinear processes, the nonlinearity created in this way does not stem from the simultaneous action of many photons, but from a population change of molecular states. Therefore, the huge nonlinearity required for optical bandwidth expansion is realized at comparatively low light intensities. Intriguingly, these intensities can be further lowered by replacing STED with a reversible optical transition that is even easier to saturate. For example, the photoswitching of optically bistable molecules typically saturates at $1-100 \mathrm{~W} / \mathrm{cm}^{2}$, which should allow for $s>$ 1000 and hence nanoscale resolution at low enough inten- sities provided by even lamps. Therefore, Eq. (2) and its verification do not only apply to STED, but also to an entire class of novel far-field light microscopes with diffractionunlimited resolution [9].

The experimental focal width of $\lambda / 48=16 \mathrm{~nm}$ can also be reduced without increasing $s$, since scaling down $\lambda_{\text {STED }}<500 \mathrm{~nm}$ readily yields values of $<10 \mathrm{~nm}$. Therefore, the resolution potential demonstrated in this work compares well with that of $8-50 \mathrm{~nm}$ in $\mathrm{x}$-ray diffraction microscopy [10], requiring a synchrotron source. Our results are in the same range with the apertureless variants of near-field optics, which, however, allow a broader range of spectroscopic studies. Using nonlinear field enhancement at a sharp tip, the apertureless near-field optics surpasses their aperture-based counterparts and reportedly attain $25 \mathrm{~nm}$ both in fluorescence [11] and in Raman scattering [12].

However, by using only focused light, our approach is neither surface bound nor does it require tight control of a nonlinearly enhanced local field that may alter the specimen. In fact, recent experiments in our laboratory have shown that the resolution potential evidenced in this study can be extended to biological fluorescence imaging. This again underscores the implication of our concept not only for optical physics, but also for the broad range of natural sciences in which optical imaging is essential.

This work has been supported by the Volkswagen Foundation.

*Corresponding author. Email: shell@gwdg.de

[1] E. Abbe, Archiv fuer Mikroskopische Anatomie und Entwicklungsmechanik 9, 413 (1873).

[2] G. Toraldo di Francia, Nuovo Cimento Suppl. 9, 426 (1952).

[3] S. W. Hell and J. Wichmann, Opt. Lett. 19, 780 (1994).

[4] A. N. Boto, P. Kok, D. S. Abrams, S. L. Braunstein, C. P. Williams, and J.P. Dowling, Phys. Rev. Lett. 85, 2733 (2000).

[5] R. Dorn, S. Quabis, and G. Leuchs, Phys. Rev. Lett. 91, 233901 (2003).

[6] T. A. Klar, E. Engel, and S. W. Hell, Phys. Rev. E 64, 066613 (2001).

[7] V. Westphal, L. Kastrup, and S. W. Hell, Appl. Phys. B 77, 377 (2003).

[8] M. Dyba and S. W. Hell, Phys. Rev. Lett. 88, 163901 (2002).

[9] S. W. Hell, Phys. Lett. A 326, 140 (2004).

[10] J. Miao, T. Ishikawa, B. Johnson, E. H. Andreson, B. Lai, and K. O. Hodgson, Phys. Rev. Lett. 89, 088303 (2002).

[11] H. G. Frey, F. Keilmann, A. Kriele, and R. Guckenberger, Appl. Phys. Lett. 81, 5030 (2002).

[12] A. Hartschuh, E. J. Sanchez, X. S. Xie, and L. Novotny, Phys. Rev. Lett. 90, 095503 (2003). 